\title{
Ultrasonic Measurement of Thicknesses of Bone and Bone Marrow within Femur Inserted with a Metal Stem*
}

\author{
Yoshihisa MINAKUCHI**, Takatoshi IDE***, Kenji TACHI**, \\ Hajime MURABAYASHI**** and Noriya AKAMATSU***
}

\begin{abstract}
The thicknesses of the bone and the bone marrow region within a pig femur inserted with a metal stem of the total hip replacement system are investigated nondestructively by means of an ultrasonic pulse echo technique. First, the wave velocities through bone and bone marrow are determined using an ultrasonic wave which enables observation of the time intervals between the first and second echoes in these specimens. Next, the ultrasonic wave is emitted toward the femur with the metal stem, which was immersed in a water tank, and the distances from a normal probe to the boundary surfaces of the bone and the metal stem are measured. Subsequently, the exact thicknesses of the bone and the bone marrow region are measured using vernier calipers. The results of the ultrasonic wave and vernier caliper measurements are graphically presented and compared. The ultrasonic method is found to be applicable for the proposed purpose.
\end{abstract}

Key Words : Biomechanics, Medical Engineering, Ultrasonic Inspection, Total Hip Replacement, Femur, Metal Stem, Bone Thickness, Bone Marrow Thickness

\section{Introduction}

Coxarthrosis and rheumatoid arthritis are becoming more common as the average age of the population becomes more advanced. Consequently, arthropathies of the exercise and load-support system are on the rise. In order to restore the function of this joint, the artificial hip joint has been widely implanted in recent years. However, relaxation, damage, wear and infection occurring after total hip replacement necessitate reimplantation. In these cases, the largest problem is the loosening between the artificial hip joint stem and the bone. An $\mathrm{X}$-ray image is mainly utilized in the diagnosis ${ }^{(1),(2)}$. However, slight loosening is difficult to distinguish in $\mathrm{X}$-ray images. When pain becomes apparent in the hip joint region, loosening

\footnotetext{
* Received 8th February, 1993. Paper No. 91-1067 A

** Faculty of Engineering, Yamanashi University, 4-311 Takeda, Kofu-shi, Yamanashi 400, Japan

*** Department of Orthopaedic Surgery, Yamanashi Medical College, 1110 Shimokato, Tamahocho, Nakakoma-gun, Yamanashi 409-38, Japan

**** Kyocera Corporation, 46-1 Takehana-donomae-cho Yamashina-ku, Kyoto 607, Japan
}

may already be so severe as to warrant replacement. Therefore, observation of the conditions inside the bone after total hip replacement, as well as the detection of slight loosening between the artificial hip joint stem and the bone, is required. Thus, the development of a measurement method is important. As one measurement method to overcome these problems, echo characteristics from the boundary surfaces of the bone and the artificial hip joint, where the sound impedance differs, are utilized. This method should reveal the conditions inside the bone. However, only a few studies have been conducted on this issue ${ }^{(3)}$.

In this work, the thicknesses of the bone and the bone marrow region within a pig femur with a metal stem are investigated nondestructively by means of an ultrasonic pulse echo technique. First, the wave velocities through bone and bone marrow are determined using an ultrasonic wave, which enables observation of the time intervals between the first and second echoes in these specimens. Next, the ultrasonic wave is emitted toward the femur with the metal stem, which is immersed in a water tank, and the distances from a normal probe to the boundary surfaces of the bone and the metal stem are measured using the linear 
scanning and circular scanning methods. Subsequently, the exact thicknesses of the bone and the bone marrow region within the femur with the metal stem are measured using vernier calipers. The results of the ultrasonic wave and vernier caliper measurements are graphically presented and compared. Thus, the utility of the proposed ultrasonic method is investigated.

\section{Measurement Principle of Ultrasonic Pulse Echo Technique}

When an ultrasonic wave arrives perpendicularly at a boundary surface between dissimilar media, part of the wave is reflected and the other part is transmitted. Then, the relationship between the sound pressures of the reflected wave $P_{r}$ and the incident wave $P_{i}$ is given as

$$
R_{p}=\frac{P_{r}}{P_{i}}=\frac{Z_{2}-Z_{1}}{Z_{1}+Z_{2}}=\frac{\rho_{2} v_{2}-\rho_{1} v_{1}}{\rho_{1} v_{1}+\rho_{2} v_{2}}
$$

where $R_{p}$ is the reflection coefficient, and $Z_{1}$ and $Z_{2}$ are acoustic impedances in media 1 and 2, respectively, which are expressed as the product of density $\rho_{i}(i=1$, $2)$ and wave velocity $v_{i}(i=1,2)$ in these media.

For diagnosis in the living body by means of the ultrasonic wave, the frequency of ultrasonic wave usually used is $1 \sim 10 \mathrm{MHz}$. In the diagnosis, echo positions of boundary surfaces where acoustical impedance differs are measured. Hence, the ultrasonic pulse echo technique is utilized to determine the conditions inside the living body.

Figure 1 shows the measurement principle of the ultrasonic pulse echo technique via immersion testing, which was used in the present experiment, where the ultrasonic wave emitted from a normal probe propagated through the part of the living body which was immersed in a water tank. The relationship between the echo height and the time is shown. Based on Fig. 1, the distance $l_{i}$ between each boundary surface is given as

$$
l_{i}=v_{i} \times \frac{t_{i}}{2} \quad(i=1,2,3, \cdots),
$$

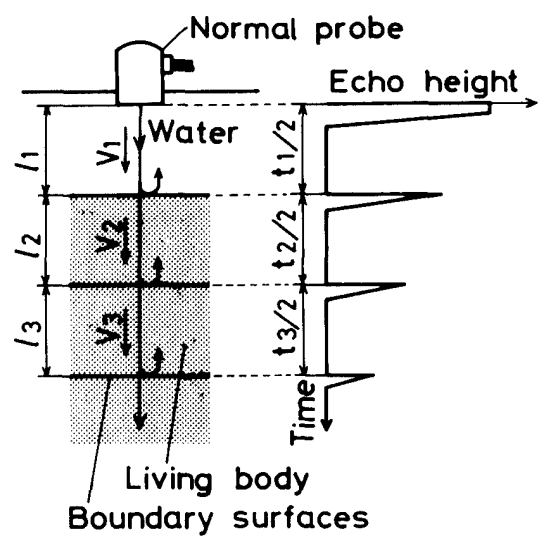

Fig. 1 Principle of ultrasonic pulse echo method where $v_{i}$ is the wave velocity and $t_{i}$ is the round-trip propagation time. Accordingly, if $v_{i}$ is a known value, $l_{i}$ is obtained by measuring $t_{i}$.

\section{Wave Velocity Measurement for Bone}

\section{1 Experimental method}

In order to measure the thickness of a bone using the ultrasonic pulse echo technique, the wave velocity through the bone must be selected beforehand. The following experiment is carried out for this purpose.

Two samples of the femur of a pig, as shown in Fig. 2, are used. Bone chips of about $2 \mathrm{~mm}$ thickness are prepared from regions (1) (5) of the midportion of the femur. Glycerin is painted onto each bone chip as a coupling medium, and a normal probe (frequency: $5 \mathrm{MHz}$, diameter of transducer: $6 \mathrm{~mm}$ ) is attached to each bone chip and pressed with a weight load of $2.3 \mathrm{~N}$. Next, the wave velocity through the bone is selected such that the thickness of the bone chip and the distance on the CRT of the ultrasonic detector agree. The gain at this time is set to $30 \mathrm{~dB}$. Furthermore, the normal probe is also used in later experiments.

\subsection{Measurement results}

Tables 1 and 2 show the measurement results of the thickness and the wave velocity in bone chips. The wave velocity through the bone chip is $2520 \sim 3000$

Table 1 Wave velocity through bone (Specimen 1)

\begin{tabular}{|c|c|c|}
\hline No. & $\begin{array}{c}\text { Thickness of } \\
\text { bone chip } \\
\text { (mm) }\end{array}$ & $\begin{array}{c}\text { Wave velocity } \\
(\mathrm{m} / \mathrm{s})\end{array}$ \\
\hline$(1)$ & 2.20 & 2860 \\
\hline (2) & 2.50 & 3000 \\
\hline (3) & 2.30 & 2720 \\
\hline$(4)$ & 2.18 & 2775 \\
\hline (5) & 2.00 & 2580 \\
\hline
\end{tabular}

Table 2 Wave velocity through bone (Specimen 2)

\begin{tabular}{|c|c|c|}
\hline No. & $\begin{array}{c}\text { Thickness of } \\
\text { bone chip } \\
\text { (mm) }\end{array}$ & $\begin{array}{c}\text { Wave velocity } \\
(\mathrm{m} / \mathrm{s})\end{array}$ \\
\hline (1) & 1.88 & 2680 \\
\hline (2) & 2.22 & 2810 \\
\hline (3) & 1.53 & 2820 \\
\hline (4) & 1.27 & 2520 \\
\hline (5) & 2.70 & 2990 \\
\hline
\end{tabular}

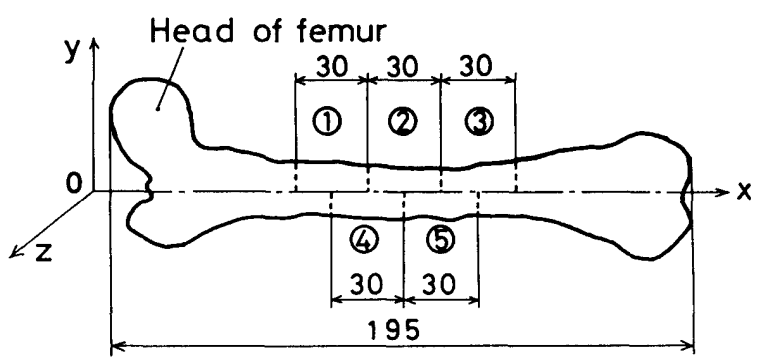

Fig. 2 Cutting position and numbering of femur 
$\mathrm{m} / \mathrm{s}$. This value changes somewhat depending on the structural condition of the bone. Therefore, the wave velocity adopted was the mean value of $2775 \mathrm{~m} / \mathrm{s}$.

\section{Wave Velocity Measurement for Bone Marrow}

\section{1 Experimental method}

In order to measure the thickness of the bone marrow region, the wave velocity through the bone marrow at body temperature must also be selected beforehand. Therefore, the following experiment is carried out.

The bone marrow within a femur is tightly sealed in an aluminum container, as shown in Fig. 3. The bone marrow is warmed to $38^{\circ} \mathrm{C}$. A normal probe is attached to the top of the aluminum container in this condition. Then, the wave velocity of the bone marrow is selected such that the thickness of the bone marrow region and the distance on the CRT of the ultrasonic detector agree. The gain at this time is set to $40 \mathrm{~dB}$.

\section{2 Measurement results}

The wave velocity through the bone marrow is $1410 \mathrm{~m} / \mathrm{s}$ at $38^{\circ} \mathrm{C}$. Furthermore, the bone marrow is comprised of liquid and jelly phases at body temperature.

\section{Thickness Measurement within Bone with Metal Stem}

\section{1 Production of measurement specimen}

In order to measure the thicknesses within the bone with the metal stem, an ultrasonic pulse echo technique via immersion testing is used, and the following specimens are produced. The femur is cut into round pieces of about $30 \mathrm{~mm}$ length from parts (1), (2) and (3), as shown in Fig. 2. The square metal stem with $12 \mathrm{~mm}$ sides or circular stem of $12 \mathrm{~mm}$ diameter is inserted into these specimens. Then, a rubber plate of

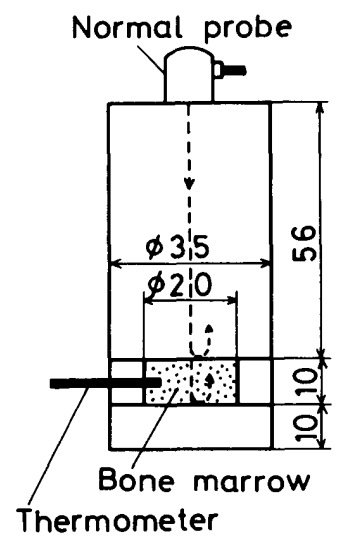

Fig. 3 Measurement device of wave velocity through bone marrow
$3 \mathrm{~mm}$ thickness is bonded to one side of the bone. Furthermore, the other side of the bone is tightly sealed using thin transparent plastic which enables observation of the inside of the bone.

Figure 4 shows the femur specimens with the square and circular metal stems. This section is observed from the condyle side. Furthermore, sides A, B, C and $\mathrm{D}$ are designated every $90^{\circ}$. The metal stem is inserted into femur specimens as parallel as possible.

\subsection{Thickness measurement within bone by lin-} ear scanning method

5.2.1 Bone with square metal stem The femur specimens in Fig. 4 are immersed in water at $38^{\circ} \mathrm{C}$. The normal probe is moved along a straight line on the surface of the water. Then, the thicknesses within the bone are measured by the linear scanning method. Figure 4 ( a ) shows the bone with the square metal stem with $12 \mathrm{~mm}$ sides positioned about $19 \mathrm{~mm}$ from the normal probe. As the normal probe moves every $1 \sim 2 \mathrm{~mm}$ toward the $z^{\prime}$ axis, echo positions from the boundary surfaces where acoustical impedance differs, are measured. The wave velocity of the ultrasonic detector is set at $1410 \mathrm{~m} / \mathrm{s}$ which is the wave velocity through bone marrow, and the gain is set to $60 \mathrm{~dB}$. Furthermore, the thicknesses within the bone are measured on the 4 sides $A \sim D$, as shown in Fig. 4 (a). Figure 5 shows the measurement device used in this experiment.

In order to verify the fundamental echo characteristic reflected from the metal stem in the linear scanning method, only the square metal stem with $12 \mathrm{~mm}$ sides is fixed at the position $19 \mathrm{~mm}$ from the normal probe, as shown in Fig. 4 (a). Then, the position of the echo reflected from the stem is measured when the normal probe is moved every $1 \mathrm{~mm}$ toward the $z^{\prime}$ axis. The wave velocity is set at $1500 \mathrm{~m} / \mathrm{s}$ which is the wave velocity through water, and the gain is set to 50 dB. Furthermore, the plane of the square metal stem

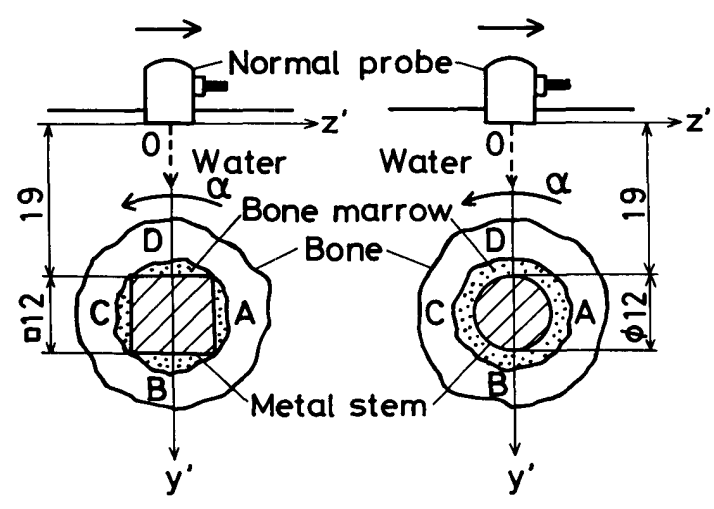

(a) Square stem

(b) Circular stem

Fig. 4 Measurement of thicknesses of bone with metal stem by linear and circular scanning methods 


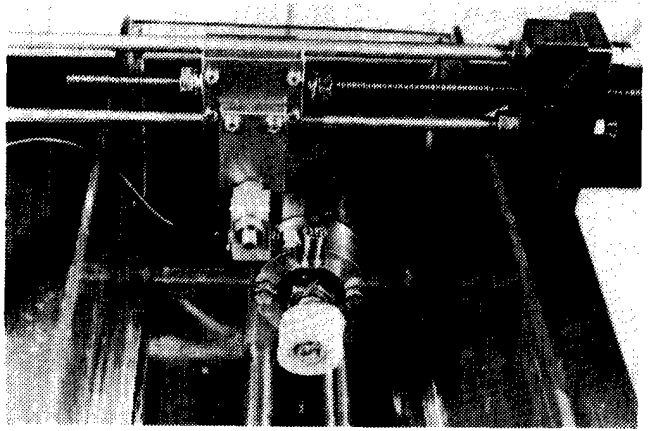

Fig. 5 Experimental device

with $12 \mathrm{~mm}$ sides is attached carefully so that the transducer face of the normal probe is as parallel as possible.

5.2.2 Bone with circular metal stem Figure 4 ( $b$ ) shows the bone with a circular metal stem of 12 mm diameter which is measured by the linear scanning method. In this case, the experiment is carried out in the same manner as described in section 5.2.1.

Also, in order to check the fundamental echo characteristic reflected from the metal stem in the linear scanning method, the experiment using only the metal stem of $12 \mathrm{~mm}$ diameter in Fig. 4 ( b ) is carried out in the same manner as described in section 5.2.1. Then, the echo position is investigated.

5. 3 Thickness measurement within bone by circular scanning method

5.3.1 Bone with square metal stem The thicknesses within the bone with the metal stems of square and circular sections are measured by the linear scanning method, as shown in Fig. 4. Figure 4 (a) shows the bone with the square metal stem with $12 \mathrm{~mm}$ sides positioned about $19 \mathrm{~mm}$ from the normal probe, which is immersed in water at $38^{\circ} \mathrm{C}$. When the normal probe is fixed and the specimen is rotated to $\alpha$ $=0^{\circ}, 90^{\circ}, 180^{\circ}$ and $270^{\circ}$, the thicknesses within the bone are measured using the echo positions reflected from the bone and the metal stem. The wave velocity of the ultrasonic detector is set at $1410 \mathrm{~m} / \mathrm{s}$ which is the wave velocity through the bone marrow, and the gain is set to $60 \mathrm{~dB}$.

5.3.2 Bone with circular metal stem Figure 4 ( b ) shows the bone which is measured by the circular scanning method. In this case, the bone specimen with the circular metal stem of $12 \mathrm{~mm}$ diameter is rotated to $30^{\circ}, 60^{\circ}, \ldots, 330^{\circ}$. The experiment is carried out in the same manner as described in section 5. 3.1.

\section{Experimental Results}

\subsection{Measurement results of thicknesses within bone}

6.1.1 Fundamental echo characteristics Figure 6 shows the measurement results of linear scan-

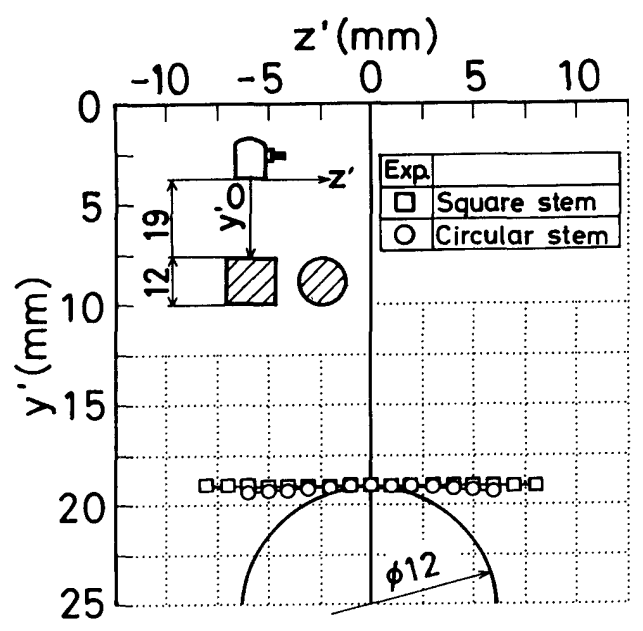

Fig. 6 Echo points from square and circular metal stems by linear scanning method

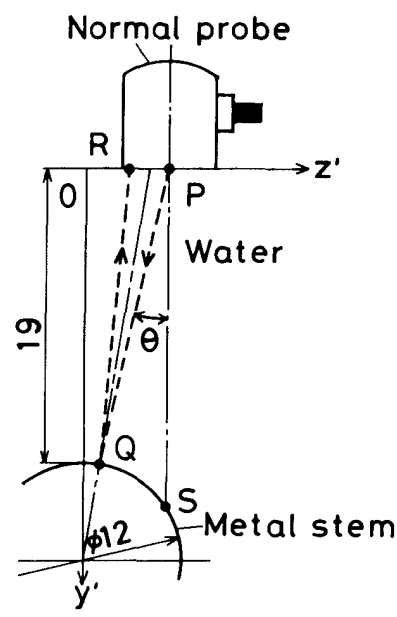

Fig. 7 Shortest propagation path of sound wave

ning method. The mark $\square$ in Fig. 6 indicates the distance from the normal probe to the square metal stem surface. If the position of the normal probe is $\left|z^{\prime}\right|$ $\leqq 8 \mathrm{~mm}$, the measured results give the same distance as the actual value. The position of $\left|z^{\prime}\right|=8 \mathrm{~mm}$ is within the measurement limit, because the domain of the sound wave received from the plane of the square metal stem is $1 \mathrm{~mm}$ and narrows greatly when the transducer diameter is $6 \mathrm{~mm}$. The mark $\bigcirc$ in Fig. 6 indicates the distance from the normal probe to the circular metal stem surface. In this case, the echo height from the metal stem surface is $\left|z^{\prime}\right| \leqq 6 \mathrm{~mm}$. The result gives a diameter fairly larger than $12 \mathrm{~mm}$. A possible cause is described below.

Figure 7 shows the shortest propagation path $\mathrm{PQR}$ of the sound wave which is reflected from the circular metal stem surface and is propagated with the beam spread angle of $\theta$. The echo obtained on the CRT indicates the position where the time is the shortest. In other words, when the normal probe is at 
point $\mathrm{P}$, the echo position received from the metal stem is not the distance of PS but is half of the path $\mathrm{PQR}$.

6.1.2 Bone with square metal stem Figure 8 shows the measurement results of the linear scanning method. The results obtained at $\mathrm{A}$ and $\mathrm{C}$ sides in the bone with the square metal stem are shown. The marks $\bigcirc, \bigcirc$ and $\square$ show the distances from the normal probe to the outside of the bone, the inside of the bone and the metal stem surface, respectively. The wave velocity of the ultrasonic detector is set at $v_{m}=$ $1410 \mathrm{~m} / \mathrm{s}$ which is the wave velocity through bone marrow. The wave velocities in bone and water are $v_{b}$ $=2775 \mathrm{~m} / \mathrm{s}$ and $v_{w}=1500 \mathrm{~m} / \mathrm{s}$, respectively. These differ from the wave velocity in bone marrow. Accordingly, the distances in bone and water are not obtained directly from the position indicated on the CRT. Therefore, these distances are modified in the following manner.

The wave velocity is set at the wave velocity $v_{m}$ in bone marrow. If the distances in bone and water indicated on the CRT are $l_{b}{ }^{\prime}$ and $l_{w}{ }^{\prime}$, respectively, the exact distances of $l_{b}$ and $l_{w}$ are given by the following equations.

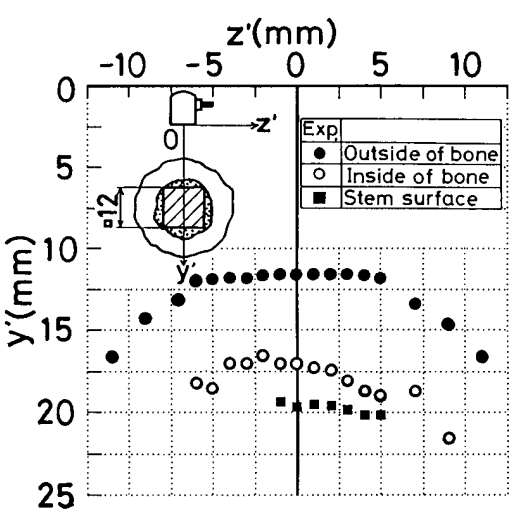

(a) A side

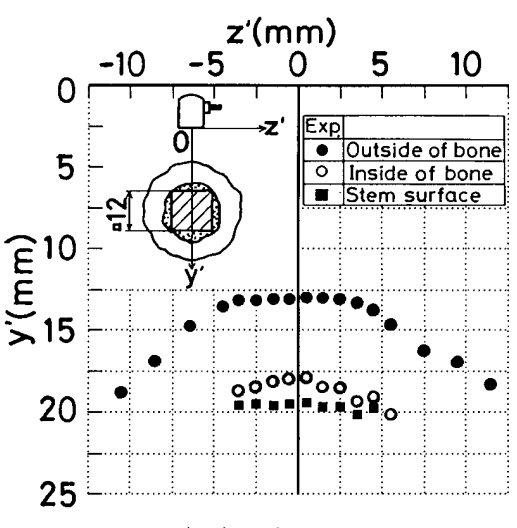

(b) C side

Fig. 8 Echo points from bone with square metal stem by linear scanning method

$$
\begin{aligned}
& l_{b}=l_{b}{ }^{\prime} \times \frac{v_{b}}{v_{m}} \\
& l_{w}=l_{w}{ }^{\prime} \times \frac{v_{w}}{v_{m}}
\end{aligned}
$$

The measurement results in Fig. 8 indicate only the points where the echo positions are exactly measured in the echoes reflected from the bone and the metal stem, because the echoes cannot be measured when the distance between the metal stem and the inside of the bone is short, since echoes from these boundary surfaces overlap each other or overlap with the delayed echoes. For these reasons, the measured points from the metal stem plane to the right and left sides of the $z^{\prime}$ axis in Fig. 8 are not the same values.

Figures 9 (a) $\sim(\mathrm{c})$ show the echo waveforms when the normal probe is at the positions of $z^{\prime}=0,3$ and $5 \mathrm{~mm}$ on the $\mathrm{C}$ side of the measured specimen. The 1st, 2nd and 3rd waveforms from the left in Fig. 9 (a) show the echo heights reflected from the outside of the bone, the inside of the bone and the metal stem surface, respectively. The 2nd and 3rd echo heights in Fig. 9 ( b ) are somewhat smaller than the corresponding echo heights in Fig. 9(a). Also, the 2nd echo height in Fig. 9(c) is almost the same as that in Fig. 9 (b). However, the 3rd echo height is fairly small. The echo height decreases as the distance between the position of $z^{\prime}=0$ and the measured point increases, and the echo position becomes unmeasurable.

Figure 10 shows the distances from the metal stem surface to the outside and inside echo positions of the bone, on the basis of the results of Fig. 8. The experimental results are indicated by $O$ and $\bigcirc$, respectively. Solid lines are the sections of the bone measured with vernier calipers. The distance from the center of the specimen to the inside of the bone can be measured with fair accuracy for positions within 6 $\mathrm{mm}$. However, slight measurement error is observed for the outside of the bone, because the inside and the outside of the bone have complicated curved surfaces. Therefore, as the distance between the center of the

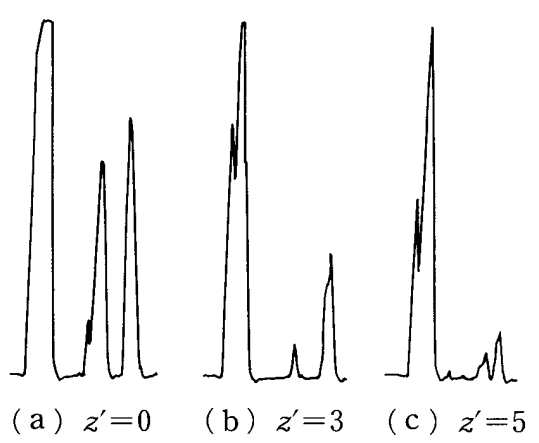

Fig. 9 Echo waveforms of bone with square metal stem at $\mathrm{C}$ side 
specimen and the measured point increases, the sound waves reflected from the outside and inside curved surfaces of the bone become difficult to detect. Moreover, the echo positions become unable to indicate the shape of the bone precisely.

\subsubsection{Bone with circular metal stem Figure}

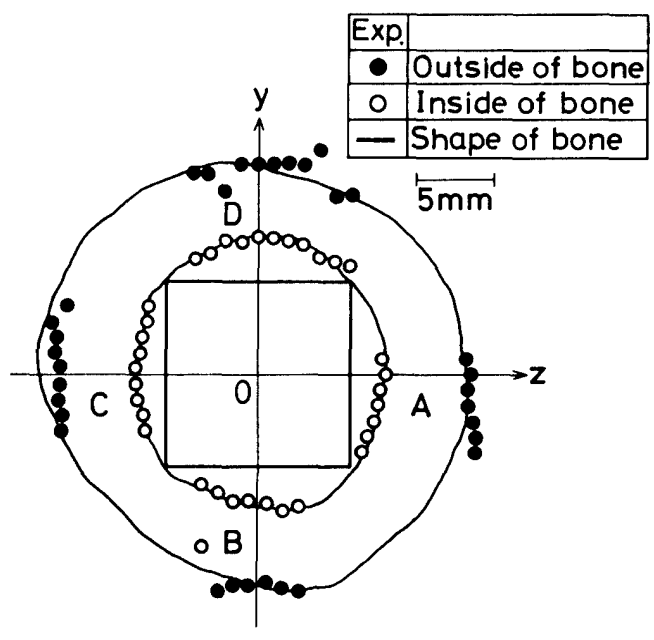

Fig. 10 Experimental results and sections of bone with square metal stem by linear scanning method

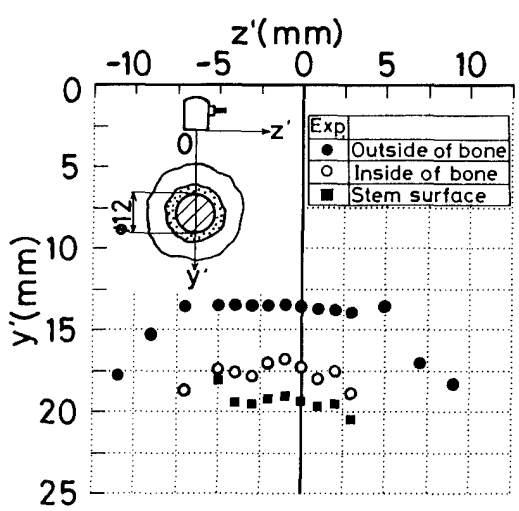

(a) A side

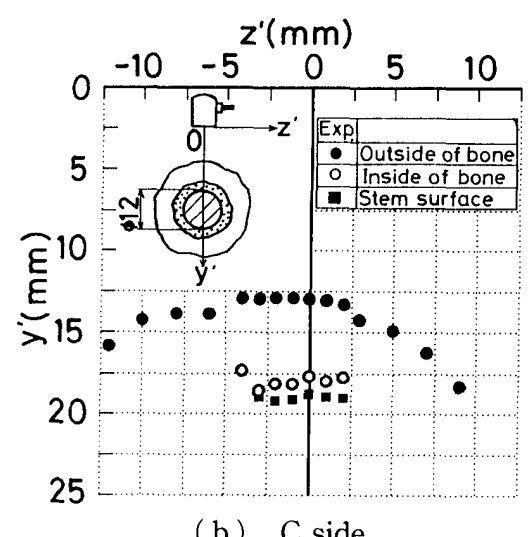

(b) C side

Fig. 11 Echo points from bone with circular metal stem by linear scanning method
11 shows the measurement results of the linear scan method. The results obtained at $\mathrm{A}$ and $\mathrm{C}$ sides in the bone with the circular metal stem are shown. The marks $\bigcirc, \bigcirc$ and $\boldsymbol{\square}$ show the distances from the normal probe to the outside of the bone, the inside of the bone and the metal stem surface, respectively. If the measured point is $\left|z^{\prime}\right| \leqq 3 \mathrm{~mm}$, the echo positions reflected from the metal stem do not change very much. Furthermore, the distances in bone and water are modified in the same manner as in section 6.1.2. In this case, it is considered that these wave velocities are $v_{b}=2775 \mathrm{~m} / \mathrm{s}$ and $v_{w}=1500 \mathrm{~m} / \mathrm{s}$, respectively. Also, the echo results for the bone and the metal stem give only the measured positions, in the same manner as in Fig. 8.

Figures $12(\mathrm{a}) \sim(\mathrm{c})$ show the echo waveforms when the normal probe is at the positions of $z^{\prime}=0$, -3 and $-5 \mathrm{~mm}$ on the A side of the measured specimen. The 1st, 2nd and 3rd waveforms from the left in Fig. 12 (a) show the echo heights reflected from the outside of the bone, the inside of the bone and the metal stem surface, respectively. The 2nd and 3rd echo heights in Fig. 12 (b) are somewhat smaller than the corresponding echo heights in Fig. 12(a). Also, the 2nd and 3rd echo heights in Fig. 12(c) are fairly small. The echo height abruptly becomes unobtainable as the distance between the position of $z^{\prime}=0$ and the measured point increases. From this figure, it is clarified that the echo position reflected from the metal stem can be measured only in the range of $\left|z^{\prime}\right| \leqq$ $3 \mathrm{~mm}$.

Figure 13 shows the distances from the metal stem surface to the outside and the inside echo positions of the bone, on the basis of the result in Fig. 11. The experimental results are indicated by $\bigcirc$ and $\bigcirc$, respectively. Solid lines are the sections of the bone measured with vernier calipers. When the normal probe is at the center of the specimen, the distances from the metal stem to the outside and the inside of the bone can be measured with fair accuracy in the center of the specimen. However, as the distance

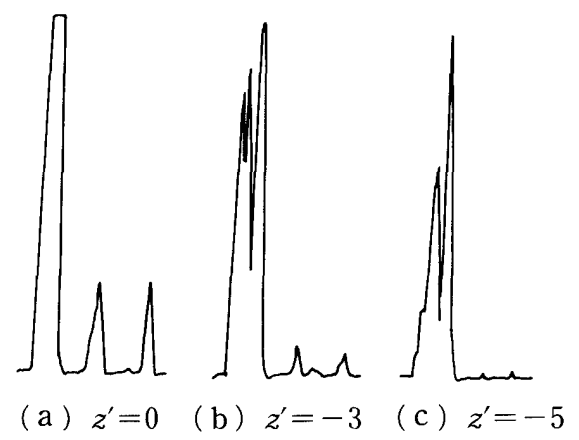

Fig. 12 Echo waveforms of bone with circular metal stem at $\mathrm{A}$ side 


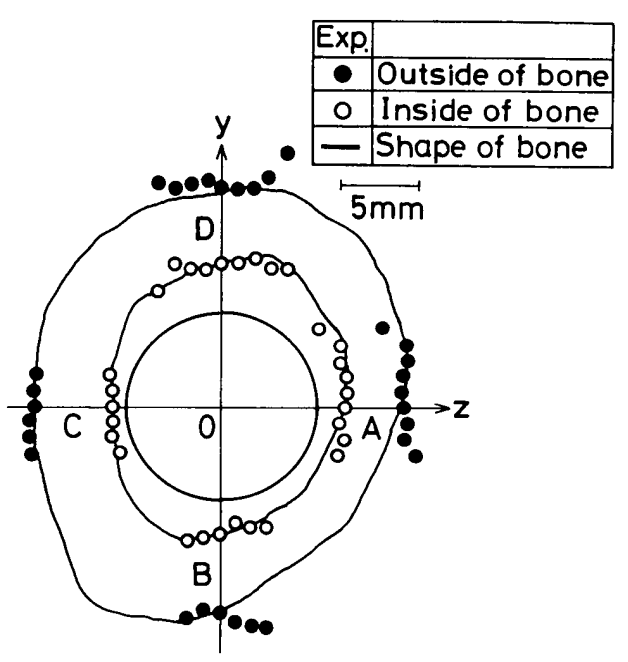

Fig. 13 Experimental results and sections of bone with circular metal stem by linear scanning method

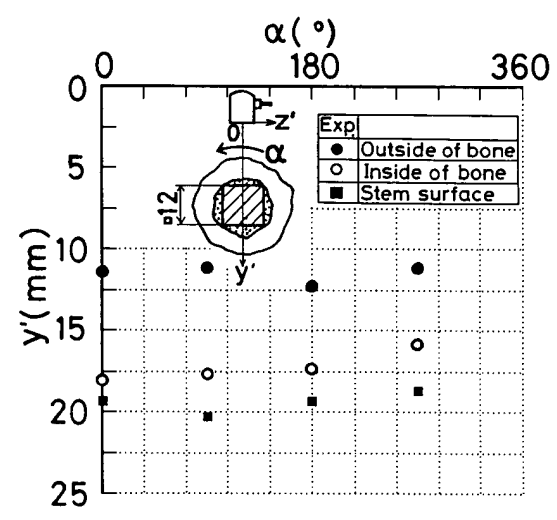

Fig. 14 Echo points from bone with square metal stem by circular scanning method

between the center of the specimen and the measured point increases, the measurement accuracy becomes poor for the outside position of the bone.

6. 2 Measurement results of thicknesses within bone by circular scanning method

6. 2. 1 Bone with square metal stem Figure 14 shows the measurement results of the circular scanning method. In this case, the specimen is rotated to $\alpha$ $=0^{\circ}, 90^{\circ}, 180^{\circ}$ and $270^{\circ}$. The marks $\bigcirc$ and $\square$ show the distances from the normal probe to the outside of the bone, the inside of the bone and the metal stem surface, respectively. Furthermore, the distances in bone and water are modified in the same manner as in section 6.1.2.

Figure 15 shows the distances at $z^{\prime}=0$ from the metal stem surface to the outside and inside echo positions of the bone, on the basis of the results in Fig. 14. The experimental results are indicated by and $\bigcirc$, respectively. Solid lines are the sections of the bone measured with vernier calipers. The thicknesses within the bone can be measured with fair accuracy.

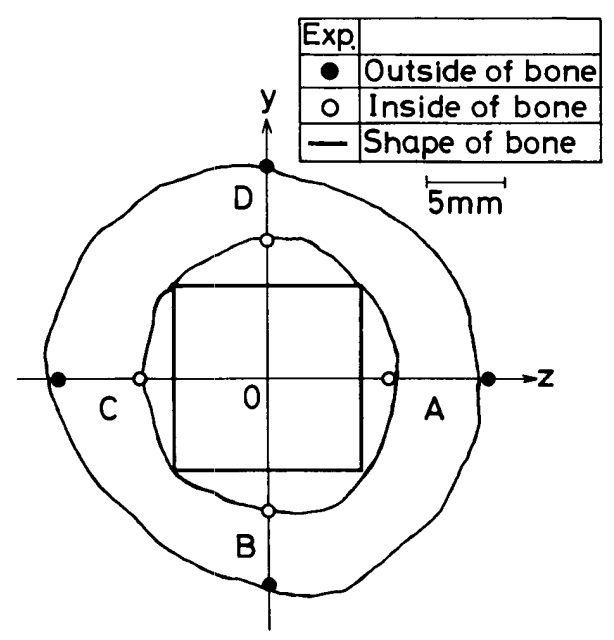

Fig. 15 Experimental results and sections of bone with square metal stem by circular scanning method

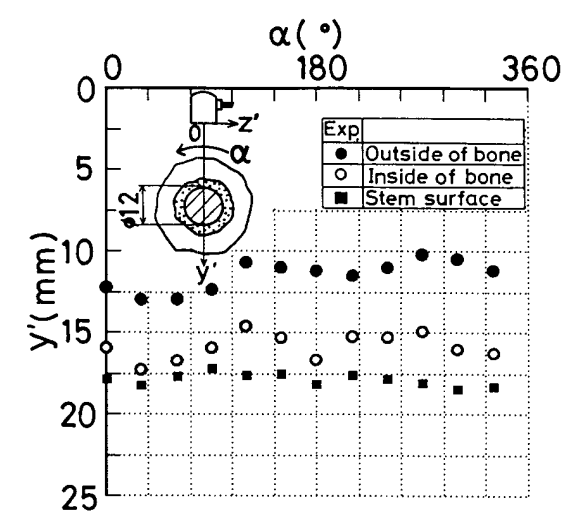

Fig. 16 Echo points from bone with circular metal stem by circular scanning method

6.2.2 Bone with circular metal stem Figure 16 shows the measurement results of the circular scanning method. In this case, the specimen is rotated to $\alpha=0^{\circ}, 30^{\circ}, 60^{\circ}, \ldots ., 270^{\circ}$. The marks $\bigcirc, \bigcirc$ and $\square$ show the distances from the normal probe to the outside of the bone, the inside of the bone and the metal stem surface, respectively. Furthermore, the distances in bone and water are modified in the same manner as in section 6.1.2.

Figure 17 shows the distances at $z^{\prime}=0$ from the metal stem surface to the outside and inside echo positions of the bone, on the basis of the result in Fig. 16. The experimental results are indicated by and $\bigcirc$, respectively. Solid lines are the sections of the bone measured with vernier calipers. The thicknesses of the bone and the distance from the inside of the bone to the metal stem can be measured with fair accuracy.

\section{Conclusions}

The thicknesses of bone and bone marrow region 


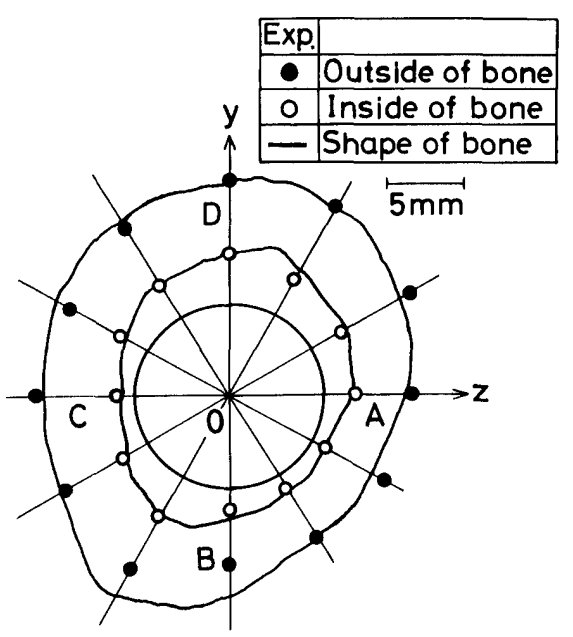

Fig. 17 Experimental results and sections of bone with circular metal stem by circular scanning method

within the femurs with square and circular metal stems are measured using the ultrasonic pulse echo technique via immersion testing. The linear and circular scanning methods are used for this measurement. For comparison, the thicknesses within the femurs are measured with vernier calipers. The results are compared and the following conclusions are obtained.

(1) The thicknesses of bone and bone marrow region within the bone with the metal stem, obtained by means of the ultrasonic method, agree fairly well with those measured using vernier calipers. The present method is found to be applicable for the proposed purpose.

(2) In the case of using the linear scanning method, if the measured point is near the center of the square metal stem, the thicknesses within the bone with the square metal stem can be measured. Additionally, if the measured point is at a position within the width of the metal stem, the distance between the metal stem and the inside of the bone can be measured in a similar manner. On the other hand, in the case of the bone with the circular metal stem, the thicknesses within the bone can be measured only near the metal stem.

(3) In the case of using the circular scanning method, if the transducer face of the normal probe is parallel to the plane of the metal stem, the thicknesses within the bone with the square metal stem can be measured. On the other hand, if the sound wave from the normal probe is emitted at the center of the metal stem, the thicknesses of the bone and the bone marrow can be measured at all positions within the bone.

The authors are deeply indebted to Professor Toshiaki Hara of Niigata University, and Professor Takashi Koizumi and Professor Toshikazu Shibuya of Tokyo Institute of Technology, for many valuable suggestions on this work.

\section{References}

(1) Smith, H. W., De Smet, A. A. and Levine, A., Measurement of Cortical Thickness in a Human Cadaver Femur (Conventional Roentgenography versus Computed Tomography), Clin. Orthop. Rel. Res., No. 169 (1982), p. 269.

(2) Meema, H. E., Cortical Bone and Osteoporosis as a Manifestation of Aging, Am. J. Roentgenol., Vol. 89, No. 6 (1963), p. 1287.

(3) Singh, S., Ultrasonic Non-destructive Measurements of Cortical Bone Thickness in Human Cadaver Femur, Ultrasonics, Vol. 27, No. 2 (1989), p. 107. 